\title{
A thermodynamic assessment of the reported room-temperature chemical synthesis of $\mathrm{C}_{2}$
}

\author{
Henry S. Rzepa (iD) ${ }^{1 凶}$
}

ARISING from K. Miyamoto et al. Nature Communications https://doi.org/10.1038/s41467-020-16025-x (2020)

T rapping experiments have recently been claimed ${ }^{1}$ to demonstrate the first chemical synthesis of the diatomic species $\mathrm{C}_{2}$ at room temperatures, using an alkynyl iodonium salt precursor. Here I explore the thermodynamic energies of the reaction using three different models, which concur in indicating that the reaction is endoenergic by $>40 \mathrm{kcal} / \mathrm{mol}$. If $\mathrm{C}_{2}$ is indeed readily formed at such temperatures, a mechanism to counter this unfavourable thermodynamic energy must be identified.

The room-temperature chemical synthesis of $\mathrm{C}_{2}$ was first reported in the form of a pre-print ${ }^{1}$ and has now appeared as a full paper ${ }^{2}$. The core of the article asserts at its simplest that a transient intermediate $\mathbf{1 1}$ formed as ${ }^{-} \mathrm{C} \equiv \mathrm{C}^{-\mathrm{I}^{+}}-\mathrm{Ph}$ by treating precursor 1a with a source of fluoride anion can fragment to singlet $\mathrm{C}_{2}$ and $\mathrm{I}-\mathrm{Ph}$ at ambient or low temperatures (Fig. 1). The so-generated $\mathrm{C}_{2}$ can then be trapped in a variety of ways which are highly suggestive of this putative intermediate. Iodonium species are indeed known in the literature as alkynylation reagents $^{3}$, albeit with a proposed mechanism of action for inserting $\mathrm{C} \equiv \mathrm{C}$ into molecules that does not involve free $\mathrm{C}_{2}$. Most of the trapping experiments in the present article are reported in solution, with an implied assertion that singlet $C_{2}$ as a discrete species is insufficiently reactive to be captured by solvent rather than by a chemical trap. One experiment is claimed to produce $\mathrm{C}_{2}$ gas, but in this case the implication is that the $\mathrm{C}_{2}$ is insufficiently reactive to be trapped by the fritted glass filter through which it must pass.

One can subject the reaction sequence in Fig. 1 to a reasonableness check based on bond dissociation energies (BDEs). A more quantitative assessment is available through higher level quantum mechanics. The authors themselves have not commented $^{2}$ on this aspect in their current article, which is based on purely experimental aspects. Addressing firstly the thermodynamics of the core equilibrium (Eq. 1)

$$
{ }^{-} \mathrm{C} \equiv \mathrm{C}-\mathrm{I}^{+}-\mathrm{Ph} \rightleftarrows \mathrm{C} \stackrel{\equiv}{=} \mathrm{C}+\mathrm{I}-\mathrm{Ph}
$$

Estimates of the experimentally derived BDE of the alkynyl-I bond in $\mathrm{C} \equiv \mathrm{C}-\mathrm{I}^{+}-\mathrm{Ar}$ iodonium salts are in the region of $70-80$ $\mathrm{kcal} / \mathrm{mol}^{4-7}$. When the iodonium C-I bond cleaves, it is directly replaced by a fourth bond as represented by $\mathrm{C} \equiv \mathrm{E}$, the $\mathrm{BDE}$ of which is experimentally estimated at the much lower value of $\sim 17$ $\mathrm{kcal} / \mathrm{mol}^{8,9}$. When allowance is made for a gain of $\sim 10 \mathrm{kcal} / \mathrm{mol}$ of free energy resulting from increase in entropy, this implies that around $43-53 \mathrm{kcal} / \mathrm{mol}$ of bond energy must be recovered by the formation at ambient temperatures of $\mathrm{C}_{2}$ itself.

To assess this aspect more quantitatively, the $\omega \mathrm{B} 97 \mathrm{XD} / \mathrm{Def} 2-$ SVPD density functional method, with solvation energies estimated using a continuum method set for dichloromethane, has been applied to the reaction shown in Fig. 1 (with $\mathrm{NMe}_{4}$ replacing $\left.\mathrm{NBu}_{4}\right)^{10}$. This suggests that the relative free energies $\Delta G_{298}$ of $\mathbf{1 a}$, 11 and the assemblage labelled " $\mathrm{C}_{2}$ " $\left(\mathrm{C}_{2}+\mathrm{I}-\mathrm{Ph}+\mathrm{Me}_{3} \mathrm{SiF}+\right.$ $\mathrm{Me}_{4} \mathrm{~N}^{+} \mathrm{BF}_{4}^{-}$) are $0.0,0.1$ and $+68.2 \mathrm{kcal} / \mathrm{mol}$, respectively. The computed energetics of $\mathrm{C}_{2}$ itself were calibrated against the two consecutive bond dissociation reactions (Eq. 2)

$$
\mathrm{HC} \equiv \mathrm{CH} \rightarrow \mathrm{HC} \equiv \mathrm{C}^{\bullet}+\mathrm{H}^{\bullet} \rightarrow \mathrm{C} \stackrel{\equiv}{=} \mathrm{C}+2 \mathrm{H}^{\bullet}
$$

for which the thermochemistry has been determined in the gas phase $^{8,9}$. This calibration suggests that the relative energy of $C \stackrel{=}{=} C$ itself is too high by $\sim 28 \mathrm{kcal} / \mathrm{mol}$ when computed using the $\omega$ B97XD DFT functional and the Def2-SVP basis set. If this correction is applied to the DFT results, then the computed free energy $\Delta G_{298}$ of the reaction $\mathbf{1 a} \rightarrow$ " $\mathrm{C}_{2}$ " is reduced from +68.2 to $\sim+40 \mathrm{kcal} / \mathrm{mol}$. This is in broad agreement with the simple argument advanced above from experimentally based BDEs $(43-53 \mathrm{kcal} / \mathrm{mol})$.

A further, simplified, model ${ }^{11}$ at the CCSD(T)/Def2-TZVPPD/ $\mathrm{SCRF}=$ dichloromethane level was computed (Eq. 3).

$$
\mathrm{Me}-\mathrm{I}^{+}-\mathrm{C} \equiv \mathrm{C}^{-} \rightarrow \mathrm{Me}-\mathrm{I}+\mathrm{C} \stackrel{\text { 兰 }}{=} .
$$

A solvation model is essential, since the ionic reactant is expected to be substantially stabilized by solvation compared to the non-ionic reaction products. At this level of theory, the energy of $\mathrm{C}_{2}$ itself is computed to be too stable by $\sim 4.6 \mathrm{kcal} / \mathrm{mol}$. With this correction applied, the overall reaction free energy emerges as $\Delta G_{298}+47.1 \mathrm{kcal} / \mathrm{mol}$, again in the range $43-53 \mathrm{kcal} / \mathrm{mol}$. The former value corresponds to a half-life of a unimolecular reaction (Eyring theory) of $\sim 10^{18}$ hours at $298 \mathrm{~K}$.

\footnotetext{
${ }^{1}$ Department of Chemistry, Molecular Sciences Research Hub, Imperial College London, White City Campus, 81 Wood Lane, London W12 OBZ, UK.

凶email: rzepa@imperial.ac.uk
} 


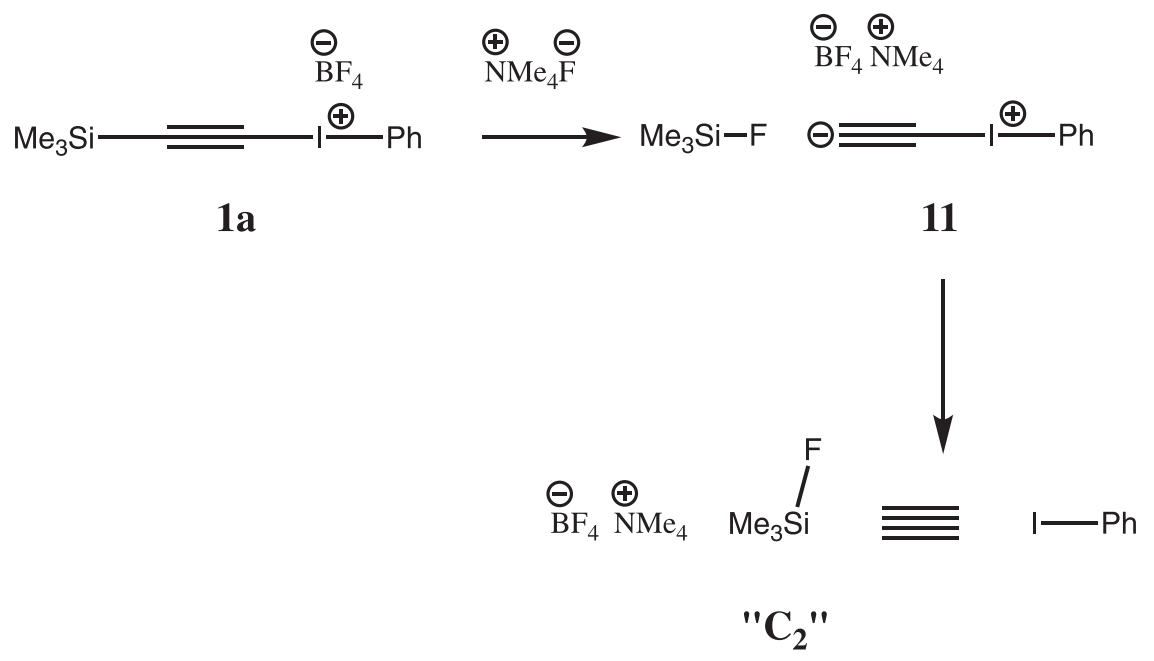

Fig. 1 Reaction scheme for proposed ${ }^{1}$ chemical synthesis of singlet $\mathbf{C}_{\mathbf{2}}$. The proposed ${ }^{1}$ chemical synthesis of singlet $C_{2}$, in which an alkynyl phenyl iodonium salt is treated with fluoride anion to remove the trimethylsilyl group and generate an internal zwitterion 11. This species is then proposed to fragment into iodobenzene and putative singlet $C_{2}$.

To add further insights, CCSD(T)/Def2-TZVPP model studies ${ }^{12,13}$ were conducted in which the $\mathrm{I}^{+}-\mathrm{Ph}$ leaving group is replaced by what must be the ultimate leaving group $\mathrm{He}^{+}$, itself formed by radioactive decay of tritium. Here, unlike the $\mathrm{C}-\mathrm{I}$ bond, the $\mathrm{BDE}$ of the $\mathrm{C}-\mathrm{He}^{+}$bond is tiny $(\sim 1 \mathrm{kcal} / \mathrm{mol})$ and its replacement by $\mathrm{C}_{2}$ does indeed then result in a reasonably exoenergic equilibrium $\left(\Delta \Delta G_{298}-42.2 \mathrm{kcal} / \mathrm{mol}\right)$, augmented again by entropy gain. This serves as a reminder that $C_{2}$ itself is a very high energy species.

How can a reaction shown in Fig. 1 and generating the proposed free $\mathrm{C}_{2}$ overcome a reaction endo-energicity $\Delta \Delta G_{298}$ of $+47 \mathrm{kcal} / \mathrm{mol}$, the most accurate estimate obtained by the computations reported here? Eyring theory tells us that at $298 \mathrm{~K}$, unimolecular reactions with respectively a half-life of $1 \mathrm{~min}$ and 1 $\mathrm{h}$ correspond to free energy barriers of 20.0 or $22.5 \mathrm{kcal} / \mathrm{mol}$, significantly lower than the range of energies predicted above. There are several possibilities to consider.

- Firstly, an as yet un-identified mechanism recovers the energy. Is it possible that sufficient enthalpy can be recovered by say reorganisation of ionic lattice energies so that the resulting free energy barrier $\Delta G_{298} \neq$ could promote a sufficiently rapid (half-life $<1 \mathrm{~h}$ ) reaction at room temperatures? This would allow $\mathrm{C}_{2}$ to be trapped by another species, but this would compete with $\sim$ barrierless reverse trapping by $\mathrm{Ph}-\mathrm{I}$. If so, by what type of mechanism could this recovered energy then be concentrated directly into the carbon-iodine bond in order to cleave it?

- That the range of thermochemical values for the reaction obtained by the three models above is wrongly predicted to be too high by $20-25 \mathrm{kcal} / \mathrm{mol}$.

- Alternatively, one might consider that free $\mathrm{C}_{2}$ itself is not produced, but instead some other species which must account for the results of the trapping reactions. A possible check on the gas-phase trapping would be to condense whatever species emerges from the dry reaction flask onto a cold-finger at liquid helium temperatures in an argon matrix and subject this directly to spectroscopic (Raman or other) analysis as an alternative to chemical trapping.

If the formation and trapping of $\mathrm{C}_{2}$ by the chemical reaction sequence shown above can indeed be independently confirmed, then this leaves us with a fascinating chemical challenge of how an otherwise apparently excessively endo-energic reaction can be promoted to viability. No solution to that perplexing dilemma is offered here.

\section{Data availability}

All relevant FAIR (Findable, Accessible, Interoperable, Reusable) datasets are available from a data repository ${ }^{10,11}$ via the collection DOIs https://doi.org/10.14469/hpc/5610 and https://doi.org/10.14469/hpc/7185 and datasets cited therein.

Received: 1 May 2020; Accepted: 21 December 2020; Published online: 23 February 2021

\section{References}

1. Miyamoto, K. et al. Room-temperature chemical synthesis of $\mathrm{C}_{2}$. ChemRxiv, Preprint at https://doi.org/10.26434/chemrxiv.8009633.v1 (2019).

2. Miyamoto, K. et al. Room-temperature chemical synthesis of $\mathrm{C}_{2}$. Nat. Commun, 11, 2134 (2020).

3. Kitamura, T. et al. Alkynylation of benzotriazole with silylethynyliodonium triflates. regioselective synthesis of 2 -ethynyl-2H-benzotriazole derivatives. J. Org. Chem. 76, 8117-8120 (2011).

4. Cottrell, T. L. The Strengths of Chemical Bonds, 2nd ed. (Butterworth,1958).

5. deB, B. Darwent, National Standard Reference Data Series. National Bureau of Standards, no. 31, Washington (1970).

6. Benson, S. W. III-Bond energies. J. Chem. Educ. 42, 502-518 (1965).

7. Kerr, J. A. Bond dissociation energies by kinetic methods. Chem. Rev. 66, 465-500 (1966).

8. Shaik, S., Danovich, D., Braida, B. \& Hiberty, P. C. The quadruple bonding in $\mathrm{C}_{2}$ reproduces the properties of the molecule. Chem. Eur. J. 22, 4116-4412 (2016).

9. Danovich, D., Hiberty, P. C., Wu, W., Rzepa, H. S. \& Shaik, S. The nature of the 4 th bond in the ground state of $\mathrm{C}_{2}$ : the quadruple bond conundrum". Chem. Eur. J. 20, 6220-6232 (2014).

10. Rzepa, H. S. Double, triple or quadruple? Does a room-temperature chemical synthesis of $\mathrm{C}_{2}$ resolve the nature of the bond?, Imp. Coll. Res. Data Reposit. https://doi.org/10.14469/hpc/5610 (2019)

11. Rzepa, H. S. A Thermodynamic assessment of the reported room-temperature chemical synthesis of $\mathrm{C}_{2}$. Imp. Coll. Res. Data Repos. https://doi.org/10.14469/ hpc/7185 (2020).

12. Rzepa, H. S. Startling bonds: revisiting $\mathrm{C} \equiv \mathrm{N}+$, via the helium bond in $\mathrm{N} \equiv \mathrm{C}$ $\mathrm{He}+$. Chemistry with a Twist (Blog), https://doi.org/10.14469/hpc/5696 (2019)

13. H. S. Rzepa, H. S. Diatomics with eight valence-electrons: formation by radioactive decay, Chemistry with a Twist (Blog), https://doi.org/10.14469/hpc/ 5736 (2019).

\section{Author contributions}

H.R. performed the calculations and wrote the text. 


\section{Competing interests}

The authors declare no competing interests.

\section{Additional information}

Correspondence and requests for materials should be addressed to H.S.R.

Peer review information Nature Communications thanks the anonymous reviewer(s) for their contribution to the peer review of this work.

Reprints and permission information is available at http://www.nature.com/reprints

Publisher's note Springer Nature remains neutral with regard to jurisdictional claims in published maps and institutional affiliations. (c) (i) Open Access This article is licensed under a Creative Commons Attribution 4.0 International License, which permits use, sharing, adaptation, distribution and reproduction in any medium or format, as long as you give appropriate credit to the original author(s) and the source, provide a link to the Creative Commons license, and indicate if changes were made. The images or other third party material in this article are included in the article's Creative Commons license, unless indicated otherwise in a credit line to the material. If material is not included in the article's Creative Commons license and your intended use is not permitted by statutory regulation or exceeds the permitted use, you will need to obtain permission directly from the copyright holder. To view a copy of this license, visit http://creativecommons.org/ licenses/by/4.0/.

(c) The Author(s) 2021 